\title{
Challenges in mathematics and statistics teaching underpinned by student - lecturer expectations
}

\author{
Deepak Parashar \\ Cancer Research Unit, Division of Health Sciences, Warwick Medical School, University of Warwick, Coventry CV4 7AL, UK \\ For correspondence: D.Parashar@warwick.ac.uk
}

\begin{abstract}
:
This study is motivated by the desire to address some of the enormous challenges faced by the students as well as the lecturer in fulfilling their respective expectations and duties demanded by the process of learning - teaching of mathematics and statistics within the framework of the constraining schedules laid down by the academic institutions and universities responsible for imparting education to students pursuing various degree programmes. The difficulties encountered in the efficient dissemination of instructions and the response of the students constitute a mechanism within which to suggest possible ways to rectify at least partially, if not wholly, the lacunae in the system and the methodology adopted by the participants engaged in this endeavour. A reasonably detailed, but by no means exhaustive, account is given of the historical developments in both mathematics and statistics primarily to arouse the interest of the students to such a level of inspiration that they draw pleasure while learning the subject. The expectations of the students as well as those of the lecturer are enumerated to a fair degree of thoroughness and possible remedial steps are suggested to minimise the differences in their perceptions using, for the first time, the famous uncertainty principle of Heisenberg, rather than employing any traditional/conventional methods that rely heavily on numerical evaluations. Wherever found essential, the scope and the goal are discussed in an environment typified by appropriate examples and similes. If followed conscientiously, the recommendations made in this paper can contribute effectively to the learning-teaching scenario with potentially significant results.
\end{abstract}

Keywords: mathematics teaching, student-lecturer expectations, uncertainty principle.

\section{Introduction}

It is universally recognised that the crucial role of mathematics in comprehending the diversity and complexity of nature has been truly hailed as one of the most robust frameworks leading to triumphs in almost all spheres of human endeavour. The unbelievable accuracy and elegance of description of various phenomena, most notably those falling in the domain of physics as also in other branches of scientific disciplines, is credited to the unambiguous language of mathematics, for the logical inquiry and academic inspiration that it inculcates. The quests for making liberal use of mathematics in the resolution of even seemingly intractable problems throws open such novel paradigms as may be deeply enchanting and truly captivating. Besides, mathematics being an indispensable tool of science and technology, it is instructive to take recourse to the ecstatic journey culminating into the present state of formulation of the subject. We, therefore, embark on a study of how mathematics as well as statistics were understood a couple of centuries ago and the stark differences with the way these are understood today. Needless to say, understanding a subject properly would require dedicated learning and effective teaching. Reflecting on the past, we focus our study on the challenges driven by the expectations of the learner and the lecturer, and how best the mathematicians and statisticians of today can produce mathematicians and statisticians of tomorrow.

This presentation is organised as follows. Sec. II is devoted to a discussion of the historical aspects of mathematics. A detailed description of the salient features that characterise its development and consequent understanding forms the central theme of this section. The historical perspective covering 
statistics is deliberated upon in Sec. III where the enormous effort that went into its formulation on firmer footings, with impeccable and mathematically sound foundations to deal with problems encountered in real situations, is accorded a place of pride that it richly deserves. In Sec. IV, we emphasise and evaluate the current scenario concerning the impact that mathematics and statistics have on the civil society, for it is the societal well-thought opinions and views that shape the progress and contribute to the development of the subject in a particularly decisive way. Sec. $\mathrm{V}$ is devoted to a discussion pertaining to the way mathematics is presently understood. It is supplemented by appending an example whereby different disciplines of mathematics are brought into the fold of a unified approach to the basic structural unity. The current status of mathematics/ statistics education is presented in Sec. VI, where a detailed mention is made of the expectations of the students and those of the lecturer. The need for continual narrowing of the apparent differences in their respective perceptions marks the main body of discussion here. Sec. VII is dedicated exclusively to the possible ways forward to achieve better compatibility by seeking tangible methods to minimise, if not completely eradicate, whatever differences exist between the students and lecturer vis-à-vis their expectations. While Sec. VIII gives a plausible description of the pedagogy of the subject, concluding remarks are appended in Sec. IX.

\section{How mathematics was historically understood and taught}

This section serves as a background to the subject for a general audience (not necessarily the fraternity of practicing mathematicians) interested in mathematics and its teaching, and provides a brief overview of mathematical understanding, which is a central theme throughout this paper.

\section{Historical perspective}

One of the greatest mathematicians of all time, Carl Frederick Gauss, expressed his sentiments through a famous statement, "Mathematics is the queen of Sciences" (von Walterhausen, 1856). To seek a plausible justification to such a claim of mathematicians to the field is by no means straightforward. Indeed the real explanation is quite complex and replete with extraordinary riddles. What actually constitutes mathematics is a matter of elaborate elucidation and even the best accounts fall short of a completely acceptable answer. All in all, "mathematics, rightfully viewed, possesses not only truth, but supreme beauty-a beauty cold and austere, like that of sculpture", as remarked in 1918 by the renowned mathematician and philosopher Bertrand Russell. Irrespective of the existence or otherwise of an accepted formal explanation, the subject reveals absolute magic and deep mystery behind some of the most significant mathematical milestones as well as the oddest objects, concepts and ideas that humankind has ever contemplated, such as those ranging from exploring subtle subatomic quantum realities and architecture of the neurons in our brains to mapping the faraway galaxies in the cosmos. Besides, it provides the grammar and dictionary of laws of thought governing the mathematical systems of logic and rationality the profound scope of which, for instance, could not have been better articulated than the eloquent statement, "At every major step, physics has required, and frequently stimulated, the introduction of new mathematical tools and concepts. Our present understanding of the laws of physics, with their extreme precision and universality, is only possible in mathematical terms", made by the famous mathematician and iconic figure, Sir Michael Atiyah.

Among the essential ingredients of the subject is the concept of abstraction which forms the basis of a comprehensive understanding of almost all aspects of mathematics in terms of a finite (and small) number of attributes. As abstraction is a very important quality and makes inroads in all different facets of mathematics, it must be acknowledged that the very first signs of abstraction emerged from the process of 'counting'. The human mind does recognize the existence of an attribute, called the 'number' (Wikipedia reference, and du Sautoy(2004)), which can be ascribed to a set or collection of entities irrespective of the nature of the constituents of this collection. The set of entities could comprise material objects like fruits in a basket or even such heterogeneous collection as birthday presents or an array of various colours of a rainbow. It might as well be even a collection of numbers 
themselves! But in order to study the working behind these sets or collections it is essential that a reasonable definition of number be properly furnished. Among the various attempts in this direction, mathematicians generally agree on the definition (which is perhaps relatively the least illogical) that involves, to start with, the two sets, say, A and B having the 'same' number of members and allowing each member of the set A to couple with a particular member of the set B and vice versa. In such a scenario, the number is defined as the totality of collections such that any two collections (e.g., the sets $\mathrm{A}$ and $\mathrm{B}$ ) in this totality have the same number of elements.

Be that as it may, the arithmetical operations with counting of numbers were dictated by considerations of necessity that had a direct bearing on business and commercial dealings. Towards this end, while totaling of stock led to the operations of 'addition' and 'multiplication', balancing of account books and sharing of commodities gave rise to 'subtraction' and 'division', respectively. The extraordinary power and elegance of the number system led Gauss to accord it a place of immense respectability by stating that the "number theory is the queen of mathematics".

A good knowledge of numbers and various operations thereof can make an indelible impact on the societal behaviour of human beings vis-à-vis their intra- and interpersonal interactions. It is precisely in this context that Benjamin Franklin in 1787 famously remarked that if statesmen had known a little more arithmetic, or were more accustomed to calculations, wars would be less frequent!

The story of geometry which is a manifestation of the spatial relationships is pretty much analogous in that its development has deep roots in ancient rituals. The Greeks were the first to have pioneered geometry and advanced a consistent framework within which to understand these spatial connections and implications thereof in an unambiguous manner. However, the fundamental role that abstraction played not only in the formulation of Euclid's version (Heath (1956), Mukunda (1997)) of geometry but also in other branches of mathematics cannot be underestimated. Needless to say, abstraction acts as an incredibly powerful sieve to preserve the ingredients that are relevant and discard those that are irrelevant. A case in point refers to the rejection of friction as irrelevant and that enabled Galileo to turn mechanics into a mathematical discipline. Euclid's postulated set of rules termed as 'axioms' were considered so sacrosanct that for any part of knowledge to be labelled as mathematical it must necessarily conform to the set of these postulates. It is gratifying to note that even the elementary arithmetic of natural numbers satisfies the stringent provisions of these axioms.

Later developments of some other mathematical areas, however, could not be accommodated in terms of the minimal set of Euclid's postulates and evidently required further elucidations. It was only in the 19th century that Dedekind (1901) addressed this problem and successfully brought calculus (invented by Newton and Leibniz) into the fold of this remarkably succinct scheme by way of extending the number system beyond the rational numbers to achieve compatibility with the postulates at least as a starting point. Based on this seminal work of Dedekind, a large body of new and perhaps even less sophisticated mathematics was derived from these very postulates of elementary arithmetic via Euclid's deductive principles. Although Newton and other mathematicians of the time dwelt on the use of Euclid's deductive reasoning, they went much beyond and made some additional assumptions the veracity of which could never be questioned.

\section{Example: Pythagoras Theorem}

(Wikipedia reference, and Raghunathan(2002))

The Pythagoras theorem states that the square of the length ' $c$ ' of the hypotenuse of a right-angled triangle is equal to the sum of the squares of the lengths of the other two sides, say, ' $a$ ' and ' $b$ ', so that $c^{2}=a^{2}+b^{2}$. If, however, the sides ' $a$ ' and ' $b$ ' are assumed to be of equal lengths and each one being of magnitude 1 , that is $a=b=1$, then it turns out that $c^{2}=2$, meaning thereby that the square of the length of the hypotenuse is of magnitude 2 . Since this ' 2 ' is a number, it looks natural to believe that the 
magnitude of ' $c$ ' should also be a number in that sense. But Pythagoras was not entirely convinced of the acceptability of this conclusion in view of the known framework of arithmetic. Since rational numbers $\mathrm{p} / \mathrm{q}$ can be constructed from the set of integers, say, $\mathrm{p}$ and $\mathrm{q}$ ( $\mathrm{q}$ being different from zero), he wondered if c could indeed be one such number. In fact, Pythagoras successfully showed that it was clearly not one of those numbers. He begins with the assumption that if $c=p / q$ and the integers $p$ and $\mathrm{q}$ have no common factors, then $2=\mathrm{c}^{2}=\mathrm{p}^{2} / \mathrm{q}^{2}$, yields the relation $2 \mathrm{q}^{2}=\mathrm{p}^{2}$. Evidently, $\mathrm{p}^{2}$ is an even number. Now since the square of an odd number is necessarily odd and that of an even is even, it is easy to conclude that $p$ must be an even number as well. One can, therefore, write $p=2 r$, with $r$ being some integer so that $\mathrm{p}^{2}=4 \mathrm{r}^{2}=2 \mathrm{q}^{2}$ so that $\mathrm{q}^{2}=2 \mathrm{r}^{2}$. This means that $\mathrm{q}^{2}$ is even and hence $\mathrm{q}$ too is even. The above consideration shows that both $\mathrm{p}$ and $\mathrm{q}$ are even numbers and admit 2 as a common factor. This is a contradiction which immediately leads to the conclusion that there exists no rational number whose square is 2 . The only way out of this enormous difficulty was simply to enlarge the number system to include numbers such as square root of 2, etc. and facilitating the application of the same kind of familiar operations working in the domain of arithmetic of rational numbers.

A viable scheme of representation of numbers of this extended system as points on a straight line added fresh impetus to the degree of flexibility to efficiently study these systems. Thus geometry transcended its conventional boundaries to embrace the extended number system within its fold. This was undoubtedly regarded as an important contribution that Pythagoras made to the progress of mathematics.

To further substantiate the wholesome nature of mathematics / statistics it is important to realise that the subject is necessarily about the study of form and structure. The preponderance of the structural relationships is clearly evident even at the basic level while dealing with the mosaic, if not outright chaos, of numbers, in that the recognition of a systematic pattern, or the 'order' constitutes a fundamental mathematical relationship which eventually leads to demystifying some of the preexisting wrong notions which were generically considered as arbitrary in the least and mysterious at the most. These relationships exist not merely between numbers but also between letters or symbols, representing the whole array of ensembles ready for mathematical analysis.

\section{Developments in Statistics}

The description of the sterling glory of mathematics would necessarily be incomplete without highlighting the enormous progress witnessed in a seemingly parallel and yet overlapping field termed as statistics. Statistics, being almost as ancient as mathematics itself (van der Waerden (1969), Dudewicz and Mishra (1988), Plaff et al (2013), Bickel and Doksum (2001), Stigler (1986, 1999), Porter (1988), Sheynin (2012)), has its roots firmly entrenched in numbers and their operations. Considered earlier as essentially a part of mathematics, statistics has now grown into a full-fledged subject in its own right. It may not perhaps be entirely inappropriate to ascribe its ascendance metaphorically to Newton's oft-quoted remark that statistics has stood on the shoulders of mathematics, so to speak. In a sense, the 'part' has come of age and has become the 'whole'. While the underlying well established principles of mathematics have been extensively used for its development, statistics has made significant inroads into extremely diverse areas of human activity. In what follows, we briefly sketch the outlines of the historical development of statistics and its various off-shoots which qualify it as a 'practical' science with enormous potential for applications to real life problems and situations.

As a term Statistics stems from the word 'State/Staatenkunde etc'. around the 1600s. So in that sense statistics can be regarded as a much younger discipline of study. Its mathematical foundations were actually laid down by the pioneers such as Pearson typically around the 1880s. One, of course, finds precursors to and notions of statistics latent in the Greek geometry where various types of mean values were defined but those were essentially geometrical rather than statistical. There are also 
biblical references to a census but then statistics as it has coherently evolved over the centuries is certainly much more than just numbers.

As a reasonable starting point, we recognise that a numerical estimate of the population and collection of information pertaining to the resources of a country could possibly have been in existence from earliest times once the relevant arithmetic involving numbers and their operations became available. There is enough historical evidence to show that the Egyptians, Babylonians and Romans maintained and preserved records of the resources of their respective states. Even in India, as far back as 300 BC, the administration of statistical data and records thereof had reached a high level of sophistication and organisation. There are numerous references to detailed statistics of various kinds of inscriptions as well as in technical treatises. In more recent times, there is ample evidence of descriptive statistics occupying an important place in the annals of records of the state. The records of abundant material so preserved contain a wealth of information, faithfully and minutely recorded in their smallest detail, with an array of facts illustrative of its extent, resources, condition, population, industry and wealth, among other things. Statistics became a subject of serious study in Europe with the rise of the modern states, notably in the $17^{\text {th }}$ and $18^{\text {th }}$ centuries. However, the development of the subject had been provided further impetus by the strident growth of economy the world over.

The development of mathematical aspects of statistics essentially commenced with the advent of the theory of probability which was formulated by Kolmogorov almost contemporaneously with the economic and official statistics. But unfortunately, these two movements remained entirely unrelated to each other and obviously oblivious of the progress in their respective domains for a long time. This was closely followed by the emergence of the theory of errors which was based on the normal distribution and pioneered by Laplace, Gauss, and other mathematicians. Although the chief application of the theory of errors was initially in the reduction of the observations in astronomy and other physical sciences, its scope was subsequently extended by Quetelet to other fields of study and research such as anthropology. Later developments included the idea of correlations discovered by Galton which found immediate applications in biological sciences, yielding immensely fruitful results.

Even though probability sprang historically from mathematics, statistics, on the other hand, is known to have had multiple origins outside the main stream of mathematics - such as the census, keeping track of populations, soldiers, and astronomy dealing with normal distributions etc. The subject was nourished over the years by intellectual giants such as Stephen Stigler $(1986,1999)$, Theodor Porter (1988), and Oscar Sheynin (2012), to name just a few.

The history of modern statistics owes its genesis, in a large measure, to Karl Pearson for his monumental contribution in the 1900s on the distribution of Chi-square. The Chi-square test provided an excellent mathematical framework by which the significance of the extent of agreement or discrepancy, for that matter, between theoretical expectations and actual observations of a measurement could be judged with incisive precision. This mechanism also lent itself for use in subsequent developments in the study of sampling distributions which served as an important ingredient to make tangible advances in statistics research such as the theory of estimation. Emboldened by the remarkable performance of the Chi-square test, Pearson was led to found a journal named Biometrika, barely a year thereafter in 1901, devoted exclusively to statistical research in problems of biological sciences. The scope of statistics was further enhanced by the seminal work of 'Student' on small samples in 1908. R. A. Fisher made phenomenal contributions to research in the field of statistics leading to subsequent developments of far-reaching consequences including the concept of maximum likelihood estimation. The precise definitions of these concepts and the exact proofs were furnished by Frechet, Rao and Lehmann and Scheffe. With his work, the subject acquired the high status that was rightfully its own. 
The methods of statistics have generated the confidence that these constitute a truly reliable and hugely convincing theoretical/mathematical framework for analysis of data for any set of observations. Armed with these tools, it is now straightforward to seek applications in other disciplines encompassing almost all branches of human activity such as mathematical, astronomical, technical, physical, chemical, actuarial, economic and financial, computational, psychological, historical, legal, physiological and pathological, hygienic and medical, biological, genetic, zoological, botanical, and agricultural, comprising a rather comprehensive list. There is hardly any area of research or study that has not been visited by statistics. It would be interesting to consider an example to judge the efficacy of statistical methods in analyzing the observed data and making credible predictions especially for the sake of better planning and future policy projections.

\section{Example: Bio-Assay}

While the enormity of the scope of statistics prevents us to deliberate on each of this vast array of different disciplines individually, we briefly highlight the case of the biological/medical sciences to serve as an illustration in view of the current flurry of research activities in this field. Let us confine ourselves to a specific aspect and discuss the case of what is technically known as Bio-Assay. Bioassay concerns itself with biological assessment of poisons and other stimuli administered to animals in varying doses. The response of the animals resulting from each of these doses given simultaneously and under similar conditions is then critically monitored and recorded. The response could be observed in the form of the mortality rate or any other behavioural pattern among them corresponding to these individual doses with a predetermined schedule of specific concentration, content and potency. The data on response rate so observed can then be plotted against the dose in order to assess the implications of the treatment in a consistent and coherent set-up. Such a graph of the response rate versus the dose, known as the dose-response diagram, would then be used to draw inference by employing various methods such as integral-approximation method, methods based on normality assumption, the method of stochastic approximation, among others. Although the conclusions arrived at by using these methods are expected, by and large, to yield a great deal of commonality, yet there exists a distinct possibility of obtaining marginally different results depending on the choice of a particular method used. The predictions may, therefore, be interpreted in broader perspective, keeping these minor statistical uncertainties in mind while formulating and scheduling further investigations.

Before concluding this discussion, a cautionary note seems appropriate. In recent years, statistics has undergone enormous changes under the impact of several factors. The generation of tremendous amounts of data of the order of terabytes in astronomical surveys and even higher amounts of data accumulated in high energy physics experiments, currently underway in Geneva, offers the possibility of implementing computations of such a huge magnitude that would have once been unthinkable. It is here that one has to exercise a judicious option regarding using proper computing techniques and programmes. Utmost care is required in handling this monumental data. No matter how sophisticated and efficient a given computer programme or algorithm may be, if the input data is flawed, the output will necessarily be flawed as well. The responsibility of a debacle like this will rest entirely with the statistician or the team of statisticians and certainly not with the statistical tools used for computation.

This section is concluded with the mention of a caveat. The material discussed in the remaining sections is essentially common to both statistics and mathematics. So wherever the word 'mathematics' appears it would automatically stand for both 'mathematics and statistics', unless a separate mention becomes absolutely indispensable and mandatory to achieve the desired clarity of the notion(s) involved. The fact that statistics is often taught in the universities/ academic institutions as a part of the mathematics curriculum goes on to emphasize the existence of a deep interrelationship between statistics and mathematics. 


\section{Impact of mathematics on civil society}

Many mathematicians digressed from the so called puritan paradigm to venture into areas that were considered relatively more pragmatic with potential for applications. This line of thought has served the humanity enormously over the years. It would be interesting to recall that since the invention of arithmetic has had its roots firmly entrenched into as diverse a field as commerce, it may well be possible that several other human activities and professions too have inspired the development of the subject and generously reaped the benefits from the resultant off-shoots in return. Thus, it became incumbent upon mathematicians of all hues to tinge their perfectionist attitude with the exigencies demanded by situations and circumstances under investigation, and seek solutions that are compatible with the observed realities. The degree of such compatibility, therefore, came to be regarded as a measure of the success as well as the usefulness of the underlying mathematics.

That is how mathematics evolved. Of course, human perceptions and circumstances have changed drastically ever since. In the modern context where business, commerce and finance play the most dominant and defining role, unfortunately the attitudes of certain sections of society and educationists towards mathematics have helped precious little. The incredible elegance and the enormous power of mathematics should not be held hostage at the behest and dictation of such forces. Instead they need to encourage and support such ventures not only for the sake of mathematics but ultimately also for their own survival. Let them know that if education costs money, so does ignorance, perhaps even more.

Unfortunately, however, mathematics does not have the advantage of being glamorous enough in comparison, for example, with other scientific disciplines such as physics, chemistry or biological sciences. This is primarily because of the tangible and ready-to-use discoveries made in these fields that have direct bearing on the society and their perceptions. Any important development in these sciences immediately catches the public attention, appreciation, and awareness. Sadly, mathematics does not enjoy this level of recognition in society even if mathematical advancements so warrant. This disparaging situation is further aggravated due to the lack of institutional support. The following example illustrates the step-motherly treatment meted out to mathematics in general.

Ironically, even Alfred Nobel did not consider important mathematical contributions to be worthy of the award of a prize. Thus mathematics was dealt a staggering blow by the very custodians who generously promoted the other sciences. To assuage, in part, the hurt-feelings and a sense of deprivation of the mathematicians in this regard, an equally prestigious prize, the Fields Medal, had subsequently been instituted in 1936 to recognise the superior achievements in mathematics, especially by young mathematicians. Regrettably, the general awareness about this medal in society remains at an abysmally low level, in contrast to the highly publicised Nobel Prize. There is, therefore, the moral duty of the society at large to accord due recognition and to work assiduously towards creating awareness about the importance and usefulness of mathematics to all segments of society in order to reap the real benefits flowing from such studies.

The following sections address these issues with special emphasis on the expectations of the students and the lecturer and comment on the effectiveness of teaching to strengthen and invigorate the subject to its level of prestige.

\section{How mathematics is presently understood}

Needless to say, mathematics has now a great opportunity to resurrect itself to usher into a truly enjoyable and exciting subject where logic prevails and brain-challenging problems accepted with pleasure and not with dismay. A large number of mathematics problems can be tackled within the 
framework of a variety of different perspectives and structures. Of course, one can exercise the option to work out the solution to a problem in a particular structure. Currently, quite a few structures/ relationships are theoretically available but making an educated, though possibly difficult, choice would largely depend on the nature of the problem for easy and unambiguous solution.

For the purpose of illustration, we might invoke either of the possible structures, namely, algebraic, geometrical, or numerical, among others, within which to seek solution of a mathematical problem. For instance, if a problem is solved algebraically, considered, a priori, less intuitively appealing rather than geometrically or numerically, it may well be due to the extreme reluctance to plot graphs or to keep track of the long list of numerical tables. Oftentimes, it may be difficult to resist the temptation to obtain the so-called nice solution but then it must come for a heavy price - compromising the rigour in favour of oversimplification. Fortunately, there exist enough mathematical tools these days so that a particular problem can be approached from all the three perspectives. This would afford a meaningful comparison of the solutions, thereby gaining relevant information and deep insights from the relationships between the three sets of solutions. These modern algorithms and techniques are contributing in a very significant way to provide a potential tool, to work with a great degree of confidence, with more realistic numbers and other mathematical formulations. Obviously, the use of these efficient and ever-evolving reliable tools, coupled with modern technology, give rise to a relationship even between different relationships.

To state this more simply, such deep connections are heading for what can be described as a grand unified theory of mathematics, suggesting intimate relation among different and apparently diverse branches such as, algebra which involves equations, and analytics involving a study of curves, among others. This unification is known as the Langlands programme which comprises a series of conjectures, which are very difficult to prove but which have influenced the course of research in the field in a big way. This programme of achieving structural unity has led Weil to initiate and shape up a new course of study and research now called algebraic geometry. There exist several other instances where this unity is equally manifest. It would be appropriate to highlight the essence of this unity through an expository example considered below.

Example: Exceptional Lie Group Es $[9,15]$

(Powell and Crasemann (1965), Cohen-Tannoudji et al (1977), Pickover (2009), Tegmark (2009), Collins(2008))

This is one of the most intriguing and perhaps the largest mathematical group known thus far for more than a century. It was considered too profound to be understood, primarily due to the lack of both mathematical ingenuity and the sufficiently advanced computational techniques. The genesis of this remarkable group can be traced to the work of the nineteenth century Norwegian mathematician, Sophus Lie, who developed a theory of continuous groups, known after him, expressing symmetries associated with the smooth rotations of object in three and higher dimensional spaces. Some simpler versions of these groups control the shape of the electron orbital symmetries as well as the symmetries of the ultimate constituents of subatomic matter called quarks. Larger groups such as the exceptional Lie group Es, suggested by the German mathematician Wilhelm Killing, is bestowed with incredible potential to help scientists understand intricate theories of physics including superstring theories and the theories of quantum gravity which are evidently the most essential ingredients towards a unified theory of all fundamental interactions in nature. The quest for taming the group Es began in 2007 with an exceptionally talented team of mathematicians and computer scientists. Elaborate and comprehensive computer software was written by the eminent Dutch computer scientist, Fokko du Clous, a member of the team, for the most powerful supercomputer available. Lo and behold, the last remaining entry in the table for Es was computed on January 8, 2007. This term describes the symmetries of a 57- dimensional object that can be thought as rotating in as many as 248 different ways without ever changing the appearance. This work constitutes a monumental advancement not 
only in mathematical knowledge but also in the harnessing of the large-scale computing techniques to obtain solutions of profound problems in mathematics. This is a classic example of structural unity of traditional mathematical knowledge and contemporary knowledge of highly technical software.

\section{How mathematics is presently taught}

It is about time that we digress from the higher echelons of developments in mathematical research to the other equally important and challenging component of imparting effective teaching in institutes of higher learning. This section is primarily intended to focus on some essential aspects of the present state of teaching and learning of mathematics at the tertiary level in the university system with special reference to the expectation of the students and those of the lecturer.

\section{Students' expectations}

A typical student's perception of the lecturer is that he is paid to teach, and the more he teaches the more the student feels he is getting his money worth. Today's students do not find it enough if the lecturer teaches the theory and concepts as outlined in the syllabus; rather, they expect the lecturer to run certain errands for them pretty much in the same manner as a travel agent makes life easier for the busy traveler such as checking and recommending flight connections, booking tickets, preparing visas, arranging hotels etc.. In particular, students tend to favour one-stop-shopping and expect the lecturer to do all the groundwork for them such as compiling easy-to-read notes, photocopying relevant sections of recommended reading material, and giving out tutorial questions that have definitive answers as opposed to open-ended ones. Such a 'Santa Claus" lecturer will certainly live up to their expectations.

Sadly, most mathematics students are not too worried about really understanding the subject. Instead, they wish only to learn the methods and tricks that will help them sail through the examinations. This is indeed a rather depressing scenario, for mathematics students already belong to a minority in the midst of other proliferating so-called lucrative options and they are easily turned off by the rigours of the subject. As a result the number of students with intrinsic liking and love for mathematics is quite small and unfortunately, even amongst them, those who eventually venture to take it up as a profession, constitute an abysmally smaller minority. The students probably feel that the beauty of mathematics can be easily appreciated and grasped if a plentiful gamut of examples having direct implications to things around them can be highlighted to them in the class room. They expect the lecturer to deliver the material at a slower pace which may be a good idea for efficiently imbibing the difficult concepts and derivations but then the stipulated topics in the course run the risk of not being completed in time. The students, however, would rejoice at this curtailment especially because they will have much fewer contents to prepare for the examinations. Evidently, the expectations of the students are extremely difficult to fulfill if the lecturer has to perform his duty conscientiously and judiciously with an eye on the completion of the course material. In some sense, this reality is puzzling and leads to a constant readjustment of the time schedule as the course progresses, since students' understanding of the subject material takes precedence over completion of the syllabus even when it is not too long. Invariably the students try to apply brakes while the lecturer strives to move forward. Needless to say, both the students and the lecturer have their own priorities and a balancing act must necessarily be performed for a successful conclusion of the course.

This is reminiscent of the famous principle of uncertainty (Heisenberg (1971)) in quantum physics the essence of which is best captured in the statement that the degree of fulfillment of the aspirations of the students is, to a large extent, at the expense of the expectations of the lecturer and vice versa. In other words, a completely satisfied lot of students will leave the lecturer in a state of complete despair. The converse of this proposition is equally true as well. A continual student-lecturer interaction with an open mind can resolve these seemingly paradoxical situations. The possibility of resolution of such problems is strongly dependent on the nurturing of a cordial relationship and rapport between the students on the 
one hand and the lecturer on the other. Therefore, the existence of a complete compatibility and mutual appreciation for each other underscores the importance of a successful conclusion of the aim of the course.

A little diversion seems appropriate at this stage. The arguments advanced herein above can be truly reinforced by taking recourse to the way the celebrated uncertainty principle of Werner Heisenberg (Nobel Laureate and regarded as a co-founder of quantum mechanics) traces its roots to a broader mathematical perspective (Powell and Crasemann (1965), Cohen-Tannoudji et al (1977), Pickover (2009)). While a detailed mathematical description lies beyond the scope of this presentation, it may just suffice to deliberate on just the essentials. For this purpose, consider a pair of dynamical variables, namely, position ' $x$ ' and momentum ' $p$ ' associated with the motion of a particle. By analogy with the standard deviation of statistics, it is customary to define the uncertainty in the variables $\mathrm{x}$ and $\mathrm{p}$ through the relations

$$
\Delta x=\left\langle(x-\langle x\rangle)^{2}\right\rangle^{1 / 2}, \quad \Delta p=\left\langle(p-\langle p\rangle)^{2}\right\rangle^{1 / 2}
$$

where the average or expectation values of $\mathrm{x}$ and $\mathrm{p}$ are defined by the relations

$$
\langle x\rangle=\int \psi^{*}(x) x \psi(x) d x, \quad\langle p\rangle=\int \psi^{*}(x)\left[-i \hbar \frac{d}{d x}\right] \psi(x) d x
$$

These uncertainties can also be equivalently expressed in terms of the concept of variance in statistics, where the squares of the uncertainties are given by

$$
\operatorname{Var}(\mathrm{x})=\mathrm{E}\left(\mathrm{x}^{2}\right)-(\mathrm{E}(\mathrm{x}))^{2}
$$

for any random variable $\mathrm{x}$. The letter $\mathrm{E}$ stands for the expectation value of the variable $\mathrm{x}$. Similar relation holds also for the conjugate variable $\mathrm{p}$. The square root of the variance is known to physicists as the standard deviation which immediately translates into uncertainties in position or momentum as outlined above.

The function $\psi(x)$ is known as the wave function of the particle and admits the statistical interpretation that $|\psi(x)|^{2} d x$ gives the probability that the position of a particle lies between $x$ and $x+d x$. Heisenberg proved that for any wave function $\psi$,

$$
\Delta x \cdot \Delta p \geq \frac{\hbar}{2}
$$

Here $\hbar=h / 2 \pi$ is the reduced Planck's constant whose value is $1.0544 \times 10^{(-27)} \mathrm{erg}$.sec. It is immediately clear that since the product of these uncertainties is always a constant irrespective of the other details of the dynamics of the particle or the system of particles, the uncertainty in the variable $\mathrm{x}$ has a reciprocal relationship with the uncertainty in the variable $\mathrm{p}$. This means that if $\Delta \mathrm{x}$ is large, then $\Delta \mathrm{p}$ must be small, and vice versa. Evidently, it is impossible to have sharp simultaneous measurement of position and momentum of a particle.

Even though the uncertainty principle has its region of validity at the micro level (atomic dimensions of the order of $10^{(-8)} \mathrm{cm}$ ), it lends itself to seek an analogy in the macro domain. In the present context, the degrees of dissatisfaction of either the students or the lecturer can be identified with those for the conjugate pair of $\mathrm{x}$ and $\mathrm{p}$ described above, and we denote these new variables by ' $\mathrm{s}$ ' for students and ' 1 ' for lecturers. Then $\Delta \mathrm{s}$ (degree of dissatisfaction of students) and $\Delta \mathrm{l}$ (degree of dissatisfaction of the lecturer) satisfy the reciprocal relationship

$$
\Delta s \propto \frac{1}{\Delta l}
$$

The consequence of the uncertainty principle, therefore, boils down to the impossibility of simultaneous fulfillment of students' aspirations and lecturer's expectations. In the light of such a paradoxical dilemma, it would make sense for university departments to include in their 
undergraduate teaching curriculum an appropriate core module addressing learning methods for mathematics students, in addition to a module for lecturers addressing teaching methods.

For the interested reader we give a further justification of the above analogy. As has been remarked, the uncertainty principle sets a limit on the precision with which certain pairs of dynamical variables such as position and momentum can be defined simultaneously. One must, however, note that there exists no theoretical limit on the accuracy of a single variable, but then the measurement of the conjugate variable disturbs the state of the system sufficiently allowing the uncertainty relation to hold. The physical implications of the Heisenberg's principle are best comprehended through a 'thought experiment', namely Heisenberg's gamma ray microscope, the salient features of which are described in detail elsewhere (e.g., Powell and Crasemann (1965)).

It is equally important to realise that the Heisenberg's uncertainty relation does hold for any other pair of conjugate variables such as time and energy $(t, e)$. In this case the relation can be expressed as

$$
\Delta e \cdot \Delta t=\hbar
$$

where $\Delta \mathrm{t}$ refers to the duration of the wave packet and $\Delta \mathrm{e}$ stands for the range of energies of the plane wave components of the wave packet under consideration. A similar relationship exists between frequency and time such that

$$
\Delta v \cdot \Delta t=1
$$

which is pretty well-known especially to electrical engineers who are, for example, aware of the fact that a one-megacycle band-width is needed to reproduce pulse of one microsecond! The uncertainty principle has been credited with enormous applications in the realm of quantum mechanics. In view of the extensive applications of the uncertainty principle, it is only appropriate to extend the simile even to the domain of human interactions. It is precisely in this context that an argument has been advanced for justifying this analogy between the students and the lecturer.

A sizeable chunk of students in the class would justifiably insist of the desirability of a well crafted lecture with utmost clarity, transparency, simplicity and lucidity. They expect the presentation to be neatly displayed either on the black board or preferably use modern technological gadgets such as laptops to project the material boldly onto the wide enough display screens so that the students face no difficulty in either seeing or taking down notes of the mathematical expressions, equations, formulas and other pertinent remarks. This arrangement, of course, requires a great deal of effort and perseverance on the part of the lecturer and must be adhered to under all circumstances to the advantage of the students.

The students expectations are, however, not limited to just learning the subject or passing an examination with good grades. They look up to the lecturer as a guide to help them decide what kinds of appropriate careers are open to them after their training as mathematicians. It is here that the role of the lecturer becomes extremely crucial in moulding the young and impressionable minds in the right directions. Students must realise that a teacher affects eternity and one can never really tell where her or his influence stops, if at all it does.

\section{Lecturer's expectations}

The professional activity of a mathematician is broadly divided mainly into teaching and research unless, of course, (s)he is also pushed into some additional administrative duties which become necessary at times in a university setup. The most important ingredient of the process of mathematics education is obviously the dissemination of instruction by the lecturer to her or his students. It is incumbent on part of the lecturer to plan the complete schedule of the course material in an unambiguous manner well in advance and set a realistic target towards this end so that the entire 
material so designed would be covered easily within the given number of lectures dictated by the duration of the academic term. (S)he is also expected to make reasonable allowance in the planned schedule for the time that might otherwise be spent by the students in activities other than academics or due to unforeseen situations beyond any one's control. The students are expected to remain alert and responsive during the lecture and a fruitful academically charged atmosphere must prevail in order for the lecturer to deliver her or his best to the students. The students must be active participants and not mere mute spectators. A good interactive session should involve frequent question-answer programme as the lecture progresses. This would automatically alleviate, at least in part, some of the apprehensions of students about the difficulty they face in the comprehension of the subject matter. The lecturer expects her or his students not only to be regular and punctual but also to be vigilant and passionate about learning the nuances of the methods of pedagogy that (s)he intends to convey with full dedication and competence. While delivering the talk it is important that the students establish an eye-to-eye contact with the lecturer so that (s)he nourishes a feeling of reassurance and resonance that the students are receptive to the salient aspects of the lecture. Such expectations of the lecturer on part of her / his students go a long way in enthusing her / him to work even harder on his lectures besides inculcating a sense of deep satisfaction at his own performance.

Since any pre-declared plan of the schedule must incorporate a time-table for periodical class tests and assignments after covering a certain portion of the syllabus, the lecturer has high expectations from his students to do well. The setting-up of the examinations has to be done with great caution and foresightedness, keeping the academic level of students in mind to enable the average student to do fairly well and the exceptionally bright ones to perform accordingly. The grading scheme of the examination must be clearly spelt out so that the students do not grudge those with comparatively high scores. The lecturer would, in this process, absolve (her//him) self of the oft-repeated charge of partiality or discrimination. Nothing hurts the lecturer more than these insinuations.

The role of the lecturer is considered sacrosanct and it is only through constant enquiry and discussion that the rough edges in understanding the relatively tough or abstract concepts can be ironed out. This exchange of ideas at the bilateral level is paramount not only to prepare the students to pursue mathematics or delve into other related domains for a career but also to face the challenges for the furtherance of the cause of mathematics education itself. There is no panacea that can be prescribed to address these challenges; and therefore a relentless struggle has to be launched to obtain concrete results towards this end. In this context, it may be worthwhile to focus attention on the message of Charles Darwin in 1876 quoted herein below:

"During the three years that I spent at Cambridge my time was wasted, as far as academic studies were concerned... I attempted mathematics, and even went during the summer of 1828 with a private tutor...but I got on very slowly. The work was repugnant to me, chiefly from my not being able to see any meaning in the early steps in algebra. This impatience was very foolish, and in after years I have deeply regretted that I did not proceed far enough at least to understand something of the great leading principles of mathematics, for men thus endowed seem to have an extra sense".

The importance insisted upon in the above beautifully-worded summary of his feelings can hardly be over-emphasised, as is borne out by the deep frustration he seems to have gone through for not having learnt the subject with due concentration, patience and dedication that it deserved. Obviously, the impending trials and tribulations in enforcing the exacting mathematical principles need first be overcome in order to achieve the stated goals. The lecturer's expectations are best realised if (s)he finds at least a significant number of her or his students with unquenchable thirst for knowledge and passion for learning the subject. It is only then that the real purpose would be served and triumphs achieved. 


\section{The way forward}

"If you give a man a fish, he will have fish to eat for just that one day; but if you teach a man to fish, he will have fish to eat for the rest of his life"

[Lao Tzu]

The above proverb is a clear reminder that education is far more beneficial to the recipient than charity!

\section{Reforming student - lecturer expectations}

Under the present circumstances, a good lecturer who is able to motivate students to life-long learning in mathematics will probably not get the good evaluation (s)he deserves. In contrast, a lecturer who panders to students' demands for a user-friendly type of teaching will probably score high marks in the students' feedback. It is, therefore, imperative that we change our students' mindset on teaching. In line with what good teaching is all about, the lecturer should probably act as a mentor offering guidance to the students, and leaving them plenty of room to explore the subject further thereby making them realise that there is no end to true learning despite the confines of the syllabus. (S)he should impress upon the students that mathematics education is meant not merely to load them with relevant mathematical principles, formulas and equations to achieve proficiency in calculations and computations, but to unsettle their minds to the level of deep analytical thought process.

In spite of the lofty expectations of both the students and the lecturer, a fair degree of flexibility and mutual trust must be exercised to move forward without sacrificing the rigour and authenticity of the subject. One must bear in mind that trust is the glue of life. It is the most essential ingredient in effective communication. It is the fundamental principle that holds all relationships. As mentioned above, each side is expected to appreciate and respect the limitations imposed by systemic considerations. A fair amount of time need be allotted for not only initiating but also encouraging frank discussion, however, trivial it may seem at first sight. Arguably, trust is not something that can be built over a coffee break or going out for lunch together; rather it is a byproduct of the successful communication between the two parties over a reasonable period of time (say first two weeks of the term, for example). The suggestions listed in the "Concluding Remarks" section implicitly include the trust building measures as a byproduct of the $1^{\text {st }}$ bullet point for Students, $3^{\text {rd }}$ bullet point for Lecturers, and the $1^{\text {st }}$ bullet point for Departments. An important thing is not to stop questioning. As has been rightly emphasised, critical appraisals and occasional disagreements often contribute to the advancement of knowledge. Once the students start developing interest in and understanding of the subject, the going could indeed be quite smooth.

The lecturer is solely responsible for projecting an image of her / his own personality and conduct so that the students have full faith and confidence in her / his expertise and scholarship of the subject besides being fair and impartial in matters as mundane as assessing their performance in the class tests or discussions. The lecturer must ensure that it is not only necessary that justice be done to her / his students but also that that justice should appear to be done. Such confidence building measures undoubtedly go a long way in fostering an ever-lasting relationship, transcending even the timebarriers of the course taught. Mathematics, with an extraordinary proliferation of ideas and (through sometimes deceptively) simple array of symbols, is perhaps the most difficult of all sciences to popularise. At times one may be compelled to choose a breezy and colloquial style of presentation and to make a judicious selection of topics that are essentially idiosyncratic in nature rather than encyclopedic, especially in the backdrop of so little time and so much to explain. This would invariably involve belabouring certain points more than others depending on their relative merits in the exercise of her/his prerogative. This is a tough balancing act but one must constantly struggle in favour of clarity in exposition as opposed to unnecessary wordiness. 
The scope of this charter does admit of some other significant, though not essentially academic, requirements to supplement the basis of a steady and more enduring interaction. The lecturer should be in full control of his temper in the event of being asked to clarify or explain even the silliest of points raised by any student. No question is too frivolous to be ignored. This necessitates a high degree of patience. Her / his dexterity in handling such complex situations may not only arouse curiosity and fascination amongst her / his students to appreciate the beauty of new emerging ideas but will also provide enough grounds to eradicate their fear of the subject. A pleasant disposition certainly reinforces this cherished relationship over and above what has been outlined herein above. The main aim of education lies in the formation of character of the students. In the words of celebrated author William Arthur Ward (Weblink reference), "a mediocre teacher tells, a good teacher explains, a superior teacher demonstrates and a great teacher inspires". This statement is particularly relevant in the context of mathematics teaching in view of the special nature of the subject.

\section{The pedagogy of mathematics teaching [11-13]}

(Zucker (1996), Holton (1998), Reid (2007))

The very essence of mathematics teaching is deeply entrenched into the roots of development of the subject itself. As stated earlier, the professional engagements of a mathematician fall into two major categories, namely teaching and research. The pursuit of each of these activities offers a great challenge and quite often encounters formidable difficulties. Mathematicians, however, regard these challenges and problems as an opportunity to delve deeper into the subject and look for appropriate solutions and plausible explanations. A successful conclusion of these challenging problems acts as a source of great pleasure for mathematicians. But anything that tends to obstruct this pleasure is sure to evoke a fair amount of frustration.

Teaching of mathematics can truly be an enjoyable experience. However, a person who dares to teach must never cease to learn. So, teaching and learning must proceed in tandem. It involves constant updating of the new findings. Reading and assimilating deep results of masters and then solving some of our own (small) problems gives us a feeling of great satisfaction like nothing else does. Since the world around us has almost a brute majority of non-mathematicians, there exist enormous challenges as also frustrations for the practitioners of mathematics if they have the basic instinct of not only surviving but also thriving in the midst of it all. The lecturer enjoys teaching if it is a course of her / his choice and the class consists of a small number of eager, motivated and well-disciplined students. But that is just a dream, for the reality is quite different from this ideal situation. On the contrary, the lecturer is often pushed into teaching large classes of uninterested students who are there just because of certain requirements that need be completed. But despite this, (s)he must strive to teach, giving her / his best without diluting the standard of the subject in any way. Compromising on rigour is absolutely inexcusable and should not, therefore, be resorted to under any circumstances. It is always preferable to prescribe a text book and a host of other relevant references to the students to consult during the progress of the course. A proper orientation must be held by the university to apprise especially the fresh entrants about the manner in which university education differs from that of the pre-university, insisting on greater independence in academic matters.

While the style of teaching varies from person to person, adherence to its basic principles and spirit remains essentially unchanged. The presentation of the lecture can, however, be fine-tuned or substantially modified depending on the response of the students but without jeopardising the importance of logical inquiry. Needless to say, all subtle aspects of mathematics including historical development, biographical sketches of great mathematicians, motivation, definitions of relevant terms, lemmas, theorems, corollaries and proofs thereof, examples, counterexamples, conjectures, constructions, computation and finally applications should find their prominent places in the scheme of things to be deliberated upon in the classroom. 
Mathematics education suffers from a peculiar dilemma though. The best researchers are concentrated either in high profile research institutes or nurture their research projects almost on a full time basis without getting an opportunity, with the exception of just a few, to share their expertise with the students at the undergraduate or post graduate levels. The students are obviously deprived of the benefits of their research. Those doing research in the forefront areas should themselves volunteer to teach a topic related to their research. This will serve as a rich source of inspiration for the budding mathematicians and also arrest the steady decline of quality of mathematics education in an effective manner.

Class tests and term examinations constitute a major part of the process of teaching. It is, therefore, extremely important to design these tests with utmost care, deep thought and innovation. The spectrum of questions should cover the entire portion of the course taught and should have a proper distribution of questions with regard to the level of difficulty. The framing of questions must keep the interest of all the students in mind. Since routine type of questions will invariably make the subject look really dull and non-enterprising for the gifted students, it is instructive that there is reasonable amount of newness in the pattern of questions. This departure from the routine is evidently expected to serve the cause of mathematics education much better.

The unrealistic portrayal of mathematics as being a difficult and dry subject is cause enough to create a sense of frustration and disgust among the mathematics fraternity. While being poor in mathematics is generally applauded, by certain sections of society, as fashionable and instinctively natural to humans, those who are good in the subject unfortunately attract comments categorising them as being queer, eccentric and crazy. Popular media and press, among others, are also partly responsible for creating this wrong public perception. The often-repeated refrain of quite a number of the so-called celebrities (the respectability associated with the word celebrity is a misnomer here; in fact they should better be referred to as nothing more than mere entertainers!) on the television channels constitutes derogatory statements like "...I just hated mathematics, my performance was truly hopeless,..." which are presented with pomp and show as if it was a great quality for others to emulate. Such unwarranted projections are bound to negatively impact the young minds and provide a dangerous recipe for avoidance of any pursuit to do mathematics. The forces that are scaring, perhaps unwittingly, the talented students away from mathematics and pulling them towards other disciplines are too powerful to be confronted. This disappointing and counterproductive scenario has already snatched away a great many would-be mathematicians. This trend must somehow be arrested and, if possible, reversed. The subject is undoubtedly like a nourishing nut that must be cracked and savored so that the insights are delivered to the students with flair and grace.

In the face of these formidable challenges, what works best in favour of mathematics is the sheer enjoyment it provides to at least some of the students who cannot even imagine of doing anything else. The question as to why mathematics is not considered enjoyable and fashionable enough at the same time has no obvious or fully satisfactory answers. Concerted efforts must be made, especially by highly renowned mathematicians, to address these issues in a non-technical manner to bring out the latent elegance of mathematics to inspire the students and apprise the laymen alike.

While lecturing to a general audience or teaching students in a class, a mathematician is always asked to intersperse his lecture by providing real life examples and illustrations as a means of motivation. Although such an expectation is not entirely without justification, yet one must appreciate that mathematics reveals its real beauty only when it is virtually disconnected from the worldly attributes. Evidently, it flourishes in the abstract. It is only when it is brought back to the realm of applications that it acquires a messy connotation. This applicable messy part of mathematics lies in the exclusive preserve of the scientists and engineers who seek solutions to real life problems. It is worth reminding one and all that mathematics is not necessarily designed to furnish only short term 
solutions but its implications could well result in making indelible imprints in the long run. Seeking any transient comfort in such a pursuit may well be a sure recipe for self-deception. A case in point is that of the computer. The basic principle on which it is based was conceived and written explicitly by George Boole (Weblink reference) almost a century earlier than its eventual discovery. Had it not been for the mathematical formulation in the form of discrete truth tables, computers would never have seen the light of the day. This is how mathematics serves the society and humanity at large.

\section{Concluding remarks}

In conclusion, through the foregoing sections one learns that mathematics cultivates not only a deep sense of perpetual wonder about the nature of mind and infinitely intriguing limits of the process of thought but also about our place in this vast universe. It is in the fitness of things to highlight the fact that the immense aura of mathematics is perceived as the pinnacle of intellectual ingenuity. The subject must, therefore, be earnestly studied and pursued with zeal in a holistic and systematic manner not only for its own sake to achieve further advances in terms of new inventions and formulations but also for rendering it compatible for use in a variety of applications in almost all spheres of human activity.

The present study highlights a spectrum of issues pertaining to the expectations of the students as well as those of the lecturer in everyday mathematics teaching. Reflecting on the past, the cultural changes in our learning and teaching methods have not necessarily advanced our understanding of the subject since most lecturers seem to pander to the students' demands instead of adhering to the needs of the profession, while most students wish to graduate with a respectable grade notwithstanding their actual level of understanding or rather the lack of it.

Much needs to be done in order to reform the current 'customer driven' style of teaching to incorporate adequate emphasis on understanding. To this extent, several suggestions have been made throughout this presentation which we summarise as follows:

Students

- must be active participants and not mere mute spectators.

- must adopt self-study methods early on in their degree course.

- must take responsibility for the level of knowledge gained during their studies.

\section{Lecturers}

- must lay enough emphasis on presentation but be careful not to overdo it lest students should take them for granted.

- must self-assess their communication skills, and be realistic about it.

- must involve frequent bilateral exchange of ideas with students not only on technical subject matter but also on learning and teaching issues.

Departments

- must initiate dialogue with entering students regarding learning methods, and perhaps including a core module for this purpose.

- must initiate dialogue with new lecturers as well as experienced ones on promoting the understanding of the uncertainty / dissatisfaction paradox mentioned earlier.

- must devise strategies to enhance communication skills of lecturers, for example, via honest and critical peer-observing colleagues during term-time lecturing.

- must deliver more public lectures to non-mathematical as well as non-academic communities to change their perception of mathematics, and bring out the latent elegance of the subject in a non-technical manner. 
A conscientious implementation and monitoring of the above programme is bound to help achieve the real goal of mathematics teaching, i.e., understanding the subject at a high level of satisfaction. Finally, all interested participants - students, lecturers, academic administrators and the institution, involved in and committed to this noble endeavour must always act in tandem and foster a cultural ambiance where mathematics flourishes through effective teaching and advancement in research for the common good of humankind.

Evidently, the role of the lecturer assumes the position of primacy in order to create fire in the belly of his pupils to be the ultimate doers, to instill in them the temperament of self-motivation fired with invincible spirits and to develop physical and mental strengths with excellence of speech, intelligence and competence to execute projects and programmes for the welfare of the community as a whole. In the discharge of this commitment and responsibility to society, the teacher is expected to expose his pupils to the latest and emerging trends of knowledge gained through a series of seminars and workshops with the participation of experts in the field. This will help evolve new, well-conceived and effective strategies to arrive at consensus solutions to problems faced by the community.

The magic, mystery and fantasy associated with this wonderful subject of mathematics are so deeply ensconced into the psyche of the human mind that a modest presentation attempted here can hardly come to a meaningful close without reflecting on the harmony, universality, and above all the supreme beauty eloquently embedded into what is appropriately described as the 'Mathematical Universe Hypothesis (MUH)' (Pickover (2009), Tegmark (2007), Collins (2008)). Following in the footsteps of stalwarts such as Kepler, Galileo, and Wigner, among others, a brilliant young mathematician, Max Tegmark, captured the real essence of mathematics in 2007 by postulating the $\mathrm{MUH}$, according to which everything in our Universe is purely mathematical. In other words, it implies that we all live within the domain of an enormously complex mathematical object, and our physical reality is indeed a mathematical structure. The profound statement that, "our Universe is not just described by mathematics - it is mathematics" encapsulates the ultimate ingredient of the MUH. Undoubtedly, the MUH may appear at first to defy conventional wisdom and intuition. But then, this must be seen in the background of many highly acclaimed and successful modern theories of physics, based on sound mathematical frameworks, such as relativity, quantum theory, and (more recently) string theory, which not only evoked ostensibly a great degree of counter-intuition when they were originally proposed and developed but had also to wade through unsavoury criticism and formidable challenges before being finally accepted as legitimate theories. It is, therefore, imperative not to succumb to the dictates of mere intuition in the larger interest of achieving the goals through lofty ideas howsoever bizarre they might seem at first. After all, the emergence of some of the most advanced theoretical formulations has been possible only through adherence to mathematical concepts and bold ideas.

We have made a modest attempt here to raise some important issues and the challenges in the overall context of mathematics education dwelling on the expectations of the students and the lecturer, and to suggest concrete remedial measures to offset the widening gap in these expectations. The endeavour has been solely to address these challenges without indulging into the conventional rhetoric and posturing. The realization of the stated goal of this work has been made possible by laying emphasis on the desirability of achieving quality rather than quantity, thereby obviating the inessential dependence on numerical evaluation. In doing so, we have based our arguments and discussion on the provisions of the celebrated uncertainty principle of Heisenberg. The exploits of this great principle, successfully used thus far only in the realm of physics with enormous applications, have been extended, perhaps for the first time, to encompass the nature of conjugation existing between the expectations of the students and the lecturer. This striking departure from the traditional methods reflects a fair degree of originality and uniqueness in providing an inspirational support and arousing 
the much needed aesthetic resonance to both the students and the lecturer alike in furthering the cause of mathematics education.

\section{Acknowledgements}

I wish to thank Prof. David Mond (Department of Mathematics) and Prof. Jane Hutton (Department of Statistics) of the University of Warwick for enlightening discussions and invaluable suggestions on a preliminary version of this paper.

\section{References}

Bickel, P.J. and Doksum, K.A. (2001). Mathematical Statistics, Vol. I. Second Edition, New Jersey: Prentice Hall. Cohen-Tannoudji, C., Diu, B. and Laloe, F. (1977). Quantum Mechanics. Vol I, New York: John Wiley \& Sons. Collins, G. (2008). Scientific American,298, p.30.

Dedekind, R. (1901). Essays on the Theory of Numbers. Chicago: Open Court Publishing Company.

Dudewicz, E.J. and Mishra, S.N. (1988). Modern Mathematical Statistics. New York: John Wiley and Sons. du Sautoy, M. (2004). The Music of the Primes. London: Harper Perennial.

Heath, T. (1956). The Thirteen Books of Euclid's Elements (3 vols). New York: Dover Publications.

Heisenberg, W. (1930). The Physical Principles of the Quantum Theory, New York: Dover Publications, p.21.

Heisenberg, W. (1971). Physics and Beyond-Encounters and Conversations. London: George Allen and Unwin.

Holton, D. (1998). ICMI Study on the Teaching and Learning of Mathematics at the University Level. Educational Studies in Mathematics 36, 91 - 103.

Mukunda, N. (1997). Current Science, 73(3), pp236-241.

Pickover, C.A. (2009). The Math Book. Sterling, New York.

Plaff, T.J., Sipos, M., Sullivan, M.C., Thompson, B.G. and Tran, M.M. (2013). Math. Magazine.

Porter, T.M. (1988). The Rise of Statistical Thinking, 1820-1900. Princeton University Press.

Powell, J.L. and Crasemann, B. (1965). Quantum Mechanics. Reading, Massachusetts: Addison-Wesley Publishing Company.

Raghunathan, M.S. (2002). Physics News, 33, p.4.

Reid, C. (2007). Hilbert. Springer.

Sheynin, O. (2012). History of Statistics. Springer Briefs in Statistics.

Stigler, S. (1986). The History of Statistics: The Measurement of Uncertainty before 1900. Cambridge, Massachusetts: The Belknap Press of the Harvard University Press; ibid. (1999).,Statistics on the Table: The History of Statistical Concepts and Methods. Cambridge, Massachusetts: Harvard University Press.

Tegmark, M. (2008). New Scientist, 195, p.39

van der Waerden, B.L. (1969). Mathematical Statistics. Heidelberg: Springer-Verlag.

von Waltershausen, W.S. (1965). Gauss zum Gedächtniss. Sändig Reprint Verlag H. R. Wohlwend (1856, reprinted 1965).

Zucker, S. (1996). Teaching at the University Level. Notices of the AMS, 43, 863-865.

http://en.wikipedia.org/wiki/Number

http://en.wikipedia.org/wiki/Pythagorean_theorem

http://www.wow4u.com/william-arthur-ward/index.html

http://www.britannica.com/EBchecked/topic/73612/George-Boole 